\title{
An Easy to Apply Methodology for Fault Detection and Isolation in Linear Systems
}

\author{
Miguel Hernandez, Basilio del Muro, Domingo Cortes, Juan Carlos Sanchez \\ National Polytechnic Institute, \\ Electrical and Mechanical Engineering School, \\ Campus Culhuacan, \\ Av. Santa Ana No. 1000, 04430, Mexico City, Mexico, \\ mahp_hernandez@hotmail.com, bdelmuro@yahoo.com, \\ domingo.cortes@gmail.com, jcsanchezgarcia@gmail.com
}

\begin{abstract}
Although the fault detection and isolation problem has been there for over three decades and many solutions using various approaches has been proposed, simpler and feasible methods to quickly and efficiently solve this problem are still valuable. In this paper, a very easy to implement method to solve the fault detection and isolation problem for linear systems is proposed. Experimental result using the three-tank system, commonly used as a benchmark, shows the feasibility of the proposed method. The technique is based on an unusual application of a long standing result of the geometric control theory for linear systems, initially proposed for the disturbance decoupling problem. Nevertheless it avoids the involved calculations that are usual in geometric-based algorithms.
\end{abstract}

Keywords: Fault Detection and Isolation, Linear Systems, Three-tank System.

\section{Introduction}

In general, a fault can be defined as a not allowed deviation in the system which leads to the inability to fulfill its intended purpose. This deviation could be in properties, characteristics or parameters which alter the system with respect to its nominal or acceptable condition [7]. To ensure the security and stability of the control system, it is important to design a feasible fault-tolerant control strategy. For any fault-tolerant strategy to work, it is necessary to detect and isolate faults quickly and effectively.

Consequently methods for detection and isolation of faults that do not affect system safety and efficiency of production are required. This represents a major challenge and is the cause why the development of techniques for Fault Detection and Isolation (FDI) has been drawn so much attention over the last three decades. The importance of the subject has led to several surveys to be published [7, 4, $5,6,8,3,19]$.

A great variety of approaches has been used to solve the Fault detection problem. Several overlapping taxonomies are used in the field usually depending on authors orientation: control engineering, mathematical, statistical or artificial intelligence. A classification that has become more or less standard is a) Data methods: Limit checking and trend checking, data analysis, spectrum analysis, pattern recognition [16] ; b) Knowledge-based methods: expert systems, fuzzy logic, neural networks [15]; c) Process model: residual generators based on state observers or parameter estimation [14]. The reader can see

the surveys previously cited, particularly [19] and the references therein for a thoroughly revision of the subject.

Although the fault detection and its related problems have been researcher for a long time the subject is far from being studied completely. On the contrary is still a very active field of research. Some examples of very recent works in the area $[12,9,1]$. On [12] an online-learning strategy is used to detect faults in a class of uncertain nonlinear system. The principal components analysis method is used to detect and localize water leakage in a water distribution network. An approach based on fuzzy models is proposed in [1] for fault detection and isolation of a compression system with some uncertain parameters.

Since the field of fault detection has become so vast, recently the research in fault detections has move from trying to solve the general problem to focus in particular applications. In [13] a solution to the FDI problem for electrical networks is proposed. The invertibility of switched system are analyzed using a set of differential equations. A solution to cope with the main source of disturbances in satellite system is proposed in [11] and an entire survey in the area is done in [18]. In [10] the parity equation method together with neural networks are used to implement a FDI system in a diesel engine. These are just some representative examples. 
Unlike most model-based FDI techniques which requires complex algorithms with involved calculations, in this paper a very easy to apply methodology for multivariable linear systems is proposed. The idea of residual generator is used, however instead of a bank of observers a single one, with a different output for each fault, is required. Furthermore, conditions under which faults can be detected are easy to check. The technique developed is based on a reinterpretation of a result proposed within the context of disturbance decoupling problem in geometric control theory (see [17]).

The problem addressed in this work is precisely described in Section 2. In Section 3 some well know results within the context of geometric control theory are presented. These results are the basis for the proposed method. In Section 4 the proposed technique is developed. It can be summarized as follows. First a fault is selected from the set of possible faults. Then, an observer to non available inputs (which happens to be the others faults) is constructed to "performs well" when faults we are not interesting in, are present. The same observer "performs bad" when the selected fault is present. Hence the residue is zero regardless other faults but becomes nonzero when the selected fault arises. These steps are repeated for each fault. Curiously the same observer can be employed for all faults just the observer output has to be changed.

Experimental application of this methodology is illustrated in Section 5 where a system consisting of three tanks interconnected by a pipe is used as a benchmark. This system has the typical characteristics of tanks, pipelines and pumps used in many industries. It has been declared as a benchmark of the COSY project of the European Science foundation. The example developed in Section 5 shows that the proposed methodology could be used as an alternative to that proposed in [9]. Finally the conclusions are given in the last Section.

\section{Problem Statement}

Consider the system

$$
\begin{aligned}
& \dot{x}=A x(t)+B u(t)+E q(t) \\
& y(t)=C x(t) \\
& z(t)=E x(t)
\end{aligned}
$$

where: $x(\cdot) \in X \mathbb{X} \mathbb{E}^{n}$ denotes the state, $u(v) \in U \mathbb{N} \mathbb{R}^{m}$ the control input, $q(v) \in Q \approx \mathbb{R}^{w}$ the disturbance input (possibly due to a system failure), $y(\cdot) \in Y \approx \mathbb{R}^{p}$ the measurable output, and $\mathbb{Z}(n) \in \mathbb{Z} \approx \mathbb{R}^{m}$ the output to be controlled; $A: X \rightarrow X, B: U \rightarrow X$, $E: Q \rightarrow X_{z} E: X \rightarrow Z$ and $C: X \rightarrow Y$ are linear applications represented by real constant matrices. Sometimes is more convenient to write (1) as

$$
\begin{aligned}
\dot{x}= & A x(t)+B u(t)+L_{1} q_{1}(t)+ \\
& +L_{2} q_{2}(t)+\ldots+L_{\eta} q_{\eta}(t)
\end{aligned}
$$

In the disturbance decoupling problem (DD), components of the vector $q=\left[\begin{array}{llll}q_{1} & q_{2} & \ldots & q_{n}\end{array}\right]$ are considered disturbances and the objective is to eliminate its influence on the system output $z(t)$. In the fault detection and isolation problem (FDI) they are treated as faults and the goal is to construct a device that indicates when $q_{t} \neq 0$. Nevertheless the two problem are closely related. In this paper a result initially proposed in the frame of DD problem is reinterpreted and adapted to solve the FDI problem. The aim of this paper is established as

FDI Probl em. Built a device (residue generator) for the system (4) that generates $a$ vector of signals

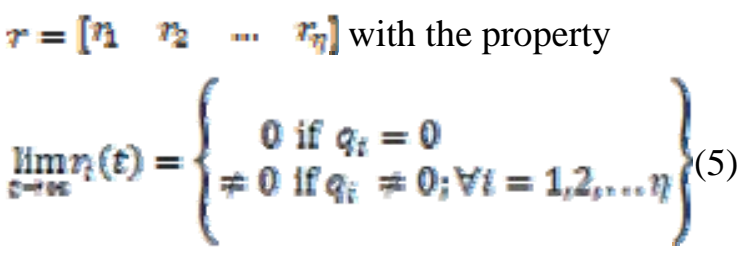

In other words, the objective of this paper is to built a device whose output is sensitive to the fault $i$-th $q_{i}(t)$, and insensitive to the others $q_{j}(t)$ for $l=1,2, \ldots, \eta_{i} j \neq t$. If this is done for every $i$ then a system that generates a warning signal in the presence of a malfunction will result.

\section{Preliminary Results}

In this section some basic results of geometric control are presented. The adaptation and reinterpretation of these results are the base for the FDI algorithm proposed. The reader can see [2] and [17] for the proof of these results. 


\subsection{A basic result for Disturbance Decoupling}

Consider the system (2). If the output $z(t)$ is insensitive to the disturbance $\mathbb{q}(t)$ then it is said that the the system is disturbance decoupled. Is clear that the system is disturbance decoupled if and only if (6) holds.

$$
\frac{z(s)}{Q(s)}=0
$$

The next theorem gives, in geometric terms, the necessary and sufficient condition for (6) to be accomplished

Lemma 1 Consider the system (2). Expression (6) holds if and only if there exist a subspace $\mathcal{J}$, A-Invariant, that is $A \mathcal{F} \subset \mathcal{T}$, such that

$$
\operatorname{ImL} \subset \mathcal{F} \subset \operatorname{ker} E
$$

\subsection{Disturbance Decoupling by Static Output Feedback (DDSF)}

If (6) does not holds but we are allowed to use state feedback, that is, to make $u=-F X$ then the problem of finding $F$ to make the closedloop system, disturbance-decoupled, is called the disturbance decoupling by state feedback problem (DDSF). Note that under state feedback the closed-loop system becomes

$\dot{x}=(A-B F) x(t)+L q(t)$.

$z(t)=E x(t)$

hence for the system $(8,9)$ to be disturbance decoupled

$$
\frac{z(s)}{\delta(s)}=E\left(s I-(A-B F)^{-1}\right) L=0
$$

must accomplish. The following result establish necessary and sufficient conditions in geometric terms for (10) to be accomplished.

Lemma 2 The DDSF problem can be solved if and only if there is $a v$ subspace, $(A-I m B)$-invariant such that

$$
\operatorname{ImE} \subset V \subset \operatorname{ker} E
$$

Note: a $V$ subspace, is $(A-I m E)$-invariant if there exist an matrix $F$ such that $\left(A-B F^{\prime}\right) v^{v} \subset \mathcal{V}$.

\subsection{Disturbance Decoupling by Output Injection (DDOI)}

An alternative to achieve disturbance decoupling is the so called static output injection. In this case the output $y(t)=C x(t)$ is injected into the system through a gain $G$. Hence the system becomes:

$\dot{x}=(A-G C) x(t)+L q(t)$.

$z(t)=E x(t)$

For $(12,13)$ to be disturbance-decoupled the expression (14) must hold.

$\frac{z(s)}{Q(s)}=E\left(s I-(A-G C)^{-1}\right) L=0$

The problem of finding $G$ such that (14) is disturbace decoupled is called the disturbance decoupling problem by static output injection (DDOI). In this case a similar result to the previous one can be established

Lemma 3 The disturbance decoupling by static injection problem can be solved if and only if there is a 5-subspace, $(\operatorname{ker} C-A)$ invariant such that

\section{ImLCSC $\operatorname{ker} \mathbb{E}$}

Note: An S-subspace is (kerc-A)-invariant if there exist a matrix $G$ such that $(A-G C) S \subset S$.

Lemma 3 can also be used to characterize the problem of reconstructing the output $z=E x(t) \quad$ under the presence of a nonaccessible $q(t)$ input. Figure 1 shows an observer of $z(t)$ given by

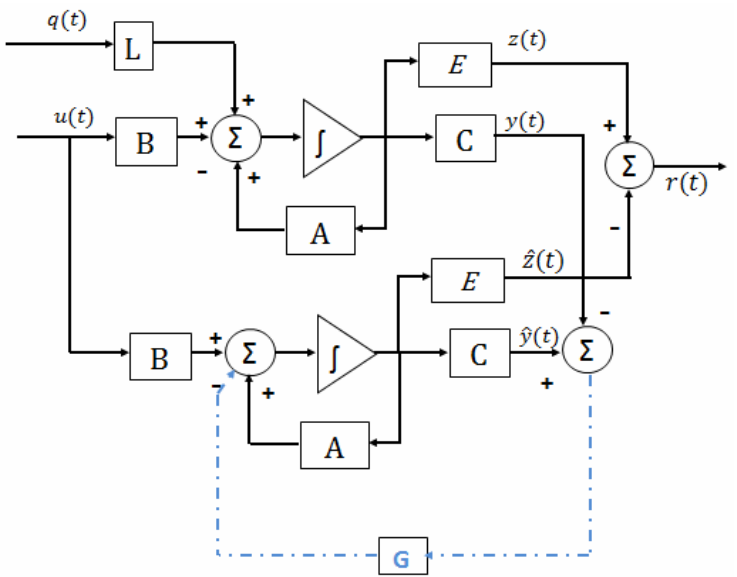

Figure 1. Observer scheme

$$
\begin{aligned}
& \hat{x}=(A-G C) \hat{x} \\
& y=C x \\
& \hat{z}=E \hat{x} \\
& r= \pm-z
\end{aligned}
$$

The goal is to design $G$ such that 


$$
\lim _{t \rightarrow=}(t)=\operatorname{Fin}_{t-w}[z(t)=z(t)]=0
$$

The next lemma establishes when this can be done. Note that the result is not trivial since signal $q(t)$ in the original system is not present in the observer.

Lemma 4 Consider the observer (16-19) (see Figure 1). There exist a matrix $G$ such that (20) holds, if and only if there is a S-subspace, (kerc-A)-invariant such that

\section{$F m E \subset S \subset \operatorname{ker} E$}

Note that conditions of Lemma 4 are the same of Lemma 3. That is the DDOI problem and the existence of the observer (16-19) are indeed equivalent.

\section{Main Results}

Lemma 4 can be used to build a fault detector by constructing an observer that is sensitive to the one fault but insensitive to others. As it will be shown below, this is achieved by designing an observer that 'performs bad' when the fault that wanted to be detected is present. Let first establish the following particular FDI problem

Particular FDI Problem (PFDI). Consider the system

$$
x=A x(t)+B u(t)+E_{\alpha} q_{a}(t)+L_{b} q_{a}(t)
$$

with $x, A, B, u$ as in system (2), $q_{a^{\prime}} q_{b} \in \mathbb{R}$ are two unknown signals (faults) and $E_{a}, E_{b}$ two vectors of $n \times 1$.

The PFDI problem is to build a residue generator of the form

$$
\begin{aligned}
& \hat{x}=(A-G C) x(t)+B u(t) \\
& r_{\alpha}(t)=t_{a}-z_{a}=E_{\alpha} x-E_{a} x \\
& r_{b}(t)=f_{b}-z_{b}=E_{b} x-E_{b} x
\end{aligned}
$$

such that

$$
\begin{aligned}
& \lim _{t \rightarrow \infty} r_{a}(t)=\left\{\begin{array}{r}
0 \text { if } q_{a}=0 \\
\neq 0 \text { if } q_{a} \neq 0
\end{array}\right\} \\
& \lim _{t \rightarrow \infty} r_{b}(t)=\left\{\begin{array}{r}
0 \text { if } q_{b}=0 \\
0 \text { if } q_{b} \neq 0
\end{array}\right\}
\end{aligned}
$$

Note that to build such device, means to propose matrices $E_{a a}, E_{b}$ and $G$ satisfying (26).
Consider first, the problem of accomplish (27). Let suppose the state is accessible. Then it can be assumed that the measurable output is $y=C x$ with $C=I_{\mathrm{w}}$. If the matrix $E_{a}$ is proposed such that $\operatorname{ker} E_{a}=\operatorname{lm} L_{b}=S$ then by Lemma 4 , an observer can be constructed such that if $q_{a}=0$ then $\lim _{\mathrm{s} \rightarrow \infty} r_{\infty}(t)=0$ regardless $q_{b}$. Furthermore, if $\operatorname{Im} L_{\sigma} \cap \operatorname{Im} L_{\sigma}=0$ then when $q_{a} \neq 0$ results in $\lim _{\varepsilon \rightarrow \infty} r_{\sigma}(t) \neq 0$. Hence, (26) is achieved. A matrix $G$ that satisfies the invariant condition $(A-G C) S \subset S$ can be easily calculated. For instance, making $A-G C=-I_{n}$, yields

$G=A+I_{n}$

Note that (28) is just an easy way to propose a matrix $G$. Different observer dynamics can be obtained by proposing $G$ differently. This completes the construction of $r_{a}$ that satisfies (26).

Construction of $r_{b}$ that satisfies (27) is straightforward following the same procedure. That is by proposing $E_{b}$ such that ker $E_{b}=I m E_{a}$ and using Lemma 4 to build an adequate observer. Matrix $G$ for this observer can be the same than that given by (28). This completes the construction of the residue generator that solve the PFDI.

Note that the assumptions made through the previous procedure were a) the state is accessible and b) $I m L_{a} \cap \operatorname{ImL} L_{b}=0$.

For seek of clarity single signals $q_{a}$ and $q_{b}$ were considered in PFDI proble $\mathrm{m}$ and its solution. However note that if $q_{a}$ and $q_{b}$ are vectors of signals the previous reasoning applies almost verbatim. Hence the following theorem has been proved.

Theorem 1 Consider the system (22) with $x$, $A, B, u$ as in system (2), $q_{\alpha} \in \mathbb{R}^{\alpha}, q_{b} \in \mathbb{R}^{g}$ are two unknown vectors of signals (faults) and $E_{a,}, E_{b}$ two vectors of $n \times \alpha$ and $n \times \beta$ respectively. Let $E_{a}$ and $E_{b}$ two matrices such that $\operatorname{ker} E_{a}=\operatorname{ImL} E_{b}$ and $\operatorname{ker} E_{b}=\operatorname{ImE} E_{a}$. If the state is accessible and $\mathrm{Im} \mathrm{L}_{\mathrm{a}} \cap \mathrm{Im} \mathrm{L}_{\mathrm{a}}=0$, then the residue generator given by (4), where $G$ is given by (28), solves the PFDI detection and isolation problem.

The following procedure summarize the solution to PFDI problem (with $q_{a t}$ and $q_{b}$ vectors).

1. Set $c=I_{m}$ and check that 


$$
I m L_{a} \cap I m E_{b}=0 \text {.Author(s) }
$$

2. Propose $E_{a}$ and $E_{b}$ such that Im $L_{b a}=\operatorname{ker} E_{\alpha}$ and $\operatorname{Im} L_{a}=\operatorname{ker} E_{b}$ Abstract

3. Set $G=A+I_{\mathrm{m}}$

Consider now the FDI problem. It can be solved by repeatedly applying the theorem 1 . For example, to generate $r_{1}$ set $q_{\alpha}=q_{1}$ and $q_{b_{b}}=\left[\begin{array}{llll}q_{z} & \mathbb{q}_{z} & = & q_{\eta}\end{array}\right]\left(L_{a}\right.$ and $L_{b}$ are set accordingly); apply the theorem 1 and set $r_{1}=r_{a}$. In general to generate $r_{i}$ set $q_{a}=q_{i}$ and $q_{t}=\left[\begin{array}{llllll}Q_{2} & \ldots & Q_{t-1} & q_{t+1} & \ldots & q_{\eta}\end{array}\right]$ apply the theorem 1 and set $r_{t}=r_{a}$.

\section{Experimental results}

The procedure developed above is applied here to a system of three interconnected tanks shown in Figure 2.

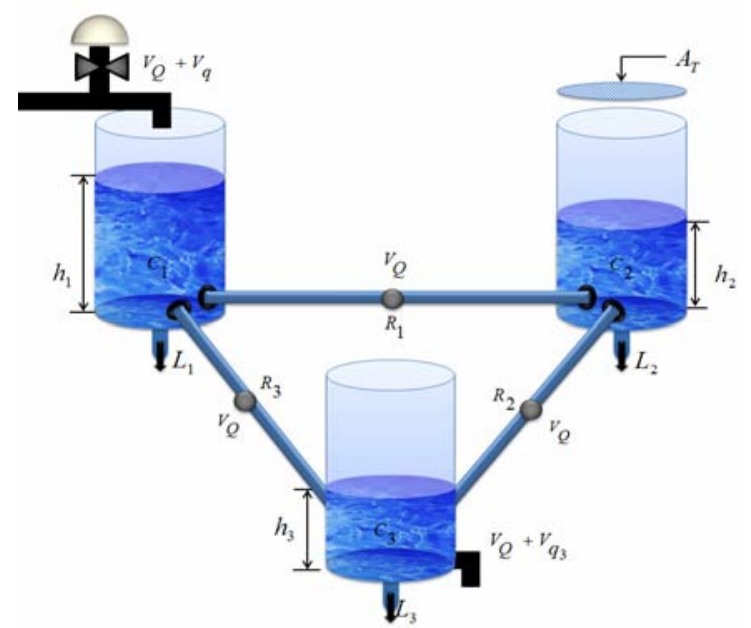

Figure 2. System of three interconnected tanks

This system has drawn much attention for research and education because on the one hand, industrial process often involves flows of liquids through interconnected tanks by pipes. On the other hand, this system exhibits characteristics that has been proven to be useful as a benchmark for testing algorithms related to parameter estimation, fault detection, fault diagnose, reconfigurable control, etc.

The plant consist of three tanks $\left(T_{1,}, T_{3}, T_{3}\right)$ typically cylindrical, with a cross-sectional area A. These cylinders are interconnected with pipes of section $s_{1,} s_{2}, s_{3}$. A liquid flows into one and is drained on the bottom of another tank. To simplify the model, a laminar flow is assumed. In this way a linear model is obtained. If the flow were turbulent, it would be still possible to obtain a linear model as long as the deviation in height and flow from their respective steady state values are small.

To describe liquid level systems in simple forms, the resistance and capacitance concepts are usually introduced. The resistance to the flow of a liquid through a pipe is defined as the change in the level needed to produce a oneunit change in flow velocity

$$
R=\frac{\text { changeintheleveldutference }(\mathrm{m})}{\text { changeintheflowrate }\left(\mathrm{m}^{\mathrm{s}} / \mathrm{sec}\right)}
$$

If the flow is laminar

$R_{V}=\frac{d B}{d V_{Q}}=\frac{H}{V_{Q}}$

with $V_{Q}=K H$. The capacitance $C$ of a tank is defined as the necessary change in the amount of stored liquid, to produce a one-unit change in the potential (level)

$$
\mathcal{C}=\frac{\text { changeinthegtonedliquild }\left(\mathrm{m}^{\mathrm{5}}\right)}{\text { changeinheight }(\mathrm{m})}
$$

To obtain a model of the three-tank system shown in Figure 2 let first to analyze the simpler system of Figure 3

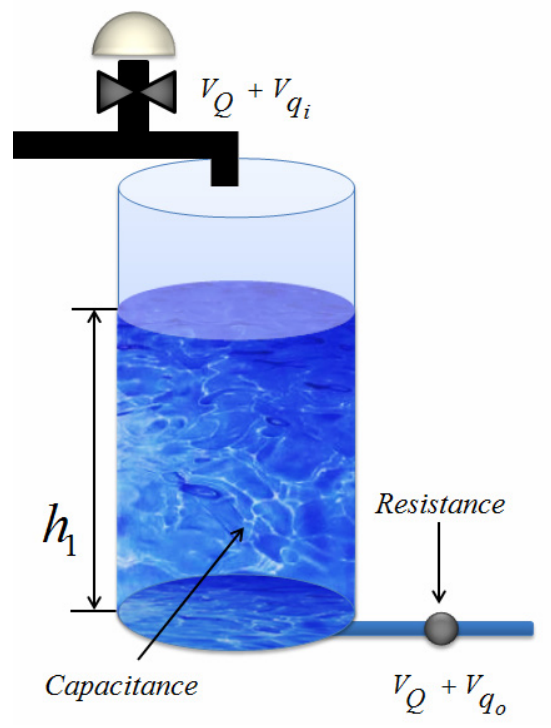

Figure 3. Simple liquid level system

with variables defined in table 1.

Table 1. Meaning of variable of Figure 3

\begin{tabular}{|c|c|}
\hline \multicolumn{2}{|c|}{ Variable Meaning } \\
\hline$V_{Q}$ & Liquid flow rate at steady state, $\left(\mathrm{m}^{3} / \mathrm{s}\right)$ \\
\hline$V_{q_{i}}$ & Input flow rate $\left(\mathrm{m}^{3} / \mathrm{s}\right)$ \\
\hline$V_{q_{o}}$ & Output flow rate $\left(\mathrm{m}^{3} / \mathrm{s}\right)$ \\
\hline$h$ & level $(\mathrm{m})$ \\
\hline
\end{tabular}


From resistance and capacitance definitions, it can be written

$$
\begin{aligned}
& \mathbb{C} d H=\left(V_{Q_{Q}}-V q_{\theta}\right) d t \\
& V q_{\theta}=\frac{\hbar}{R}
\end{aligned}
$$

Then

$$
R \mathcal{R} \frac{d h}{d z}+h=R V_{Q_{h}}
$$

If $q_{i}$ is considered as the input and $V_{q_{\Omega}}$ is taken as the output, from (34) the transfer function results

$$
\frac{V_{Q_{e}}(s)}{V_{Q_{i}}(s)}=\frac{1}{R_{s}+1}
$$

Extending the previous analysis to system shown in Figure 2, the block diagram of Figure 4 can be obtained. Note that the same crosssection area is considered for each tank. The cylindrical pipes are also considered equal. From the block diagram in Figure 4.
It consist in three small cylindrical recipients interconnected by pipes. System parameters values are specified in Table 2.

Table 2. Physical parameters of the actual system

\begin{tabular}{|c|c|}
\hline Parameter & Value \\
\hline$h_{1}$ & $0.144 m$ \\
\hline$h_{2}$ & $0.13 m$ \\
\hline$h_{3}$ & $0.116 m$ \\
\hline$A_{T}$ & $0.062 m$ \\
\hline
\end{tabular}

Table 3. Technical data for the system modeling

\begin{tabular}{|c|c|}
\hline Parameter & Value \\
\hline$V_{Q}$ & $0,000004 \mathrm{~m}^{\mathrm{s}} / \mathrm{s}$ \\
\hline$V_{Q_{i}}$ & $0,0015 \mathrm{~m}^{\mathrm{s}} / \mathrm{s}$ \\
\hline $\mathcal{C}_{1}=\mathscr{C}_{2}=\mathcal{C}_{3}$ & $0,0052 \mathrm{~m}^{2}$ \\
\hline$R_{1}$ & $72000 \mathrm{~m} / \mathrm{m}^{3} / \mathrm{s}$ \\
\hline$R_{Z}$ & $65000 \mathrm{~m} / \mathrm{m}^{3} / \mathrm{s}$ \\
\hline$R_{3}$ & $58000 \mathrm{~m} / \mathrm{m}^{3} / \mathrm{s}$ \\
\hline
\end{tabular}

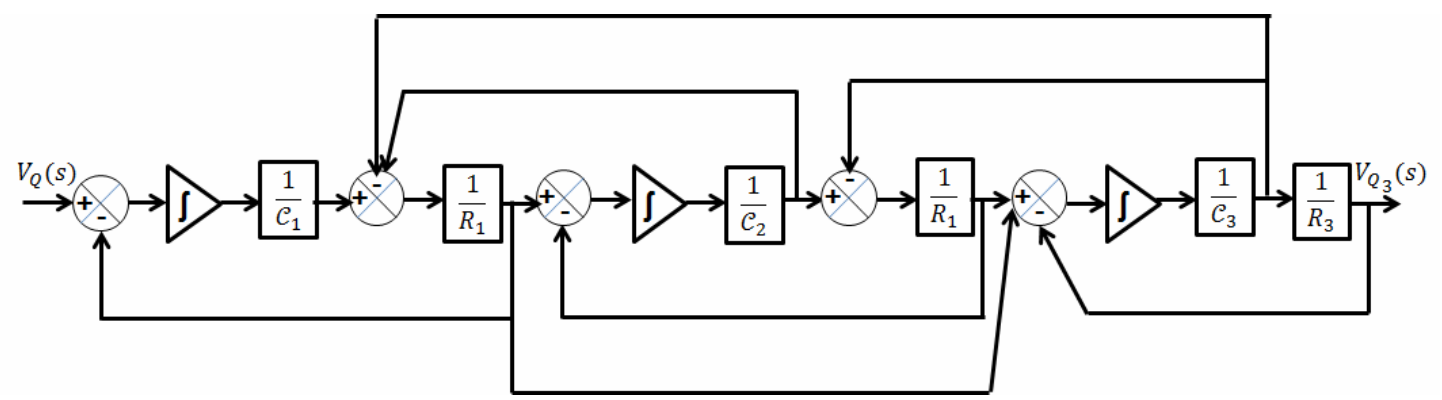

Figure 4. Block diagram of the System of Liquid Level of three tanks

The following state-space representation can be obtained

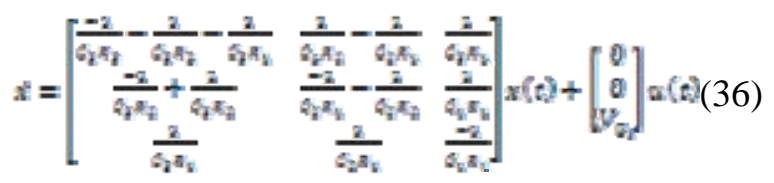

The actual system built in the lab is shown in Figure 5.

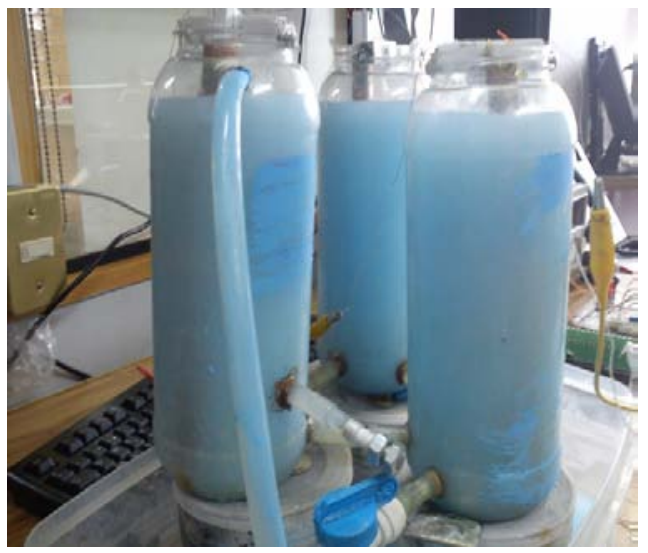

Figure 5. Actual system built in the lab
These values yield to resistances and capacitances given in Table 3. Substituting these parameters in (36) results in the model (37)

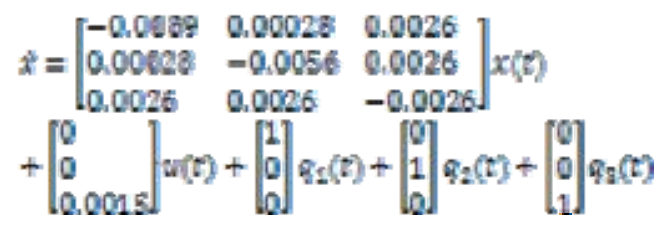

Having the model (37), the results in Section 4 can be applied. Since the three levels are measured, the state is accessible. To detect and isolate the fault $q_{1}$, set $q_{\Phi_{\mathrm{s}}}=q_{1}$ and $q_{b_{2}}=\left[\begin{array}{ll}q_{2} & q_{8}\end{array}\right]$, that is $L_{a_{8}}=L_{1}$ and $L_{b_{11}}=\left[\begin{array}{ll}L_{2} & E_{g}\end{array}\right]$. It is easy to check that ${ }^{\prime} m L_{c_{2}} \cap F_{m} E_{b_{2}}=0$. Then theorem 1 is applicable. Following the procedure previously established results in

$$
E_{1}=\left[\begin{array}{lll}
1 & 0 & 0
\end{array}\right]
$$

and 


\section{$G=\left[\begin{array}{lll}0,9991 & 0,0002 & 0,0026 \\ 0,0002 & 0,9943 & 0,0026 \\ 0,0026 & 0,0026 & 0,9973\end{array}\right]$}

To detect an isolate $q_{2}$ set $q_{\sigma_{z}}=q_{2}$ and $q_{u_{2}}=\left[\begin{array}{ll}Q_{1} & q_{3}\end{array}\right]$ that is $E_{\sigma_{2}}=L_{2}$ and $L_{u_{t_{2}}}=\left[\begin{array}{ll}E_{1} & L_{2}\end{array}\right]$.

Consequently $\quad\left[m L_{\Sigma_{2}} \cap \operatorname{Im} \mathbb{L}_{\dot{E}_{2}}=0\right.$. Applying again theorem 1 results in

$$
E_{2}=\left[\begin{array}{lll}
0 & 1 & 0
\end{array}\right]
$$

For $q_{3}, E_{\sigma_{s}}=E_{3}$ and $L_{b_{x}}=\left[\begin{array}{ll}E_{1} & L_{2}\end{array}\right]$, then $I m L_{a_{\mathrm{y}}} \cap E m L_{b_{\mathrm{s}}}=0$ and application of theorem 1 results in

$$
E_{0}=\left[\begin{array}{lll}
0 & 0 & 1
\end{array}\right]
$$

The complete residue generator is given by

$$
\begin{aligned}
& \hat{x}=(A-G C) x(t)+B u(t) \\
& r_{1}(t)=E_{1}(\hat{x}-x) \\
& r_{2}(t)=E_{2}(x-x) \\
& r_{3}(t)=E_{3}(x-x)
\end{aligned}
$$

with $G_{s} E_{1}, E_{2}, E_{2}$ are given by (38-41).

The residue generator given by (42-45) was implemented in a PC. The level sensors were made of wire resistors. Its measurements were fed to the PC using an A/D board.

Figure 6 shows the error between the estimated states $x$ and the measured states $x$ when there is no faults. In can be seen that the observer given by (42) performs well.

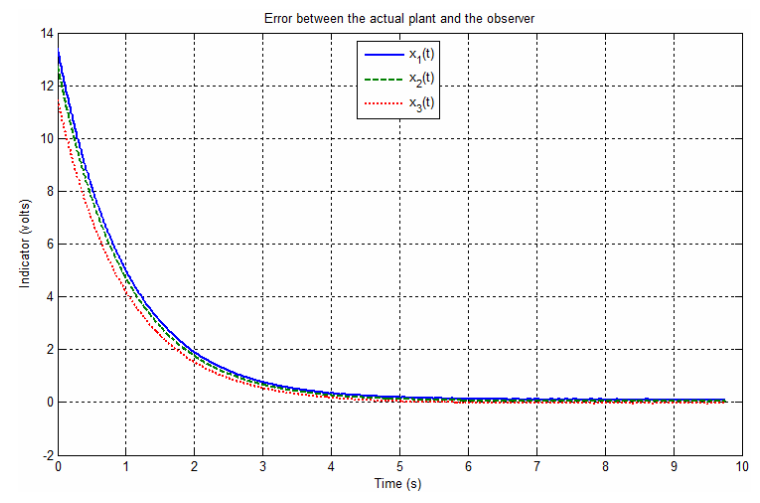

Figure 6. Error between the real system and the observer

In Figure 3, after the tanks are in the stationary level and the errors of the observers are cero a fault $q_{3}$ is simulated by opening a faucet in tank 3 (at $t=1.8 \mathrm{sec}$ ). As shown in Figure 7 as soon as the fault is present, the signal $r_{3}$ start to grow, when this signal reach a given threshold a warning signal is triggered by the PC software. The same happens if fault $q_{1}$ or $q_{2}$ are introduced.

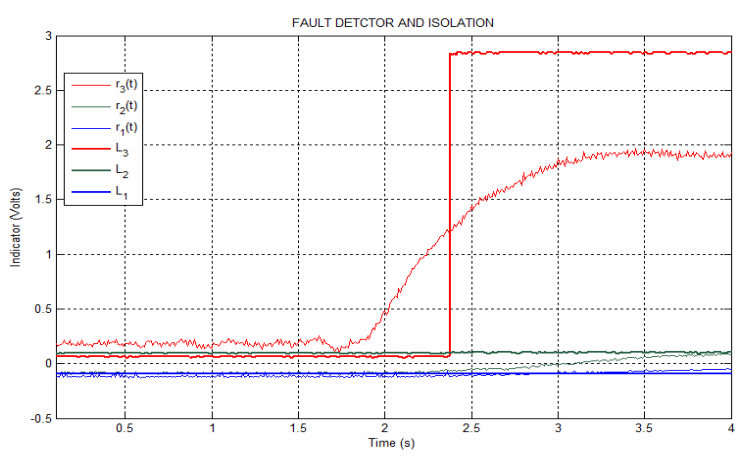

Figure 7. Performance of the experimental residue generator

\section{Conclusions}

A new technique for solving the fault detection and isolation when the state is accessible has been proposed. The condition under which this technique can be applied is expressed in very simple terms. While the proposed methodology is based on geometric control theory it is surprisingly simple to apply avoiding the sophisticated calculations that are so common in geometric-based algorithms. This new technique is based on a novel application of the theory of asymptotic observers to non accessible inputs.

The typical benchmark consisting in interconnected tanks has been used to illustrate the application of the method. Although no formal analysis has been done, experimental results show that the the residue generator is robust under reasonable modeling errors. Furthermore, is easy to implement and calibrate and it requires low computational effort. By its simplicity, speed of response, and straightforward implementation the FDI solution that has been proposed could be used in some fault-tolerant control strategies.

\section{REFERENCES}

1. HAFAIFA, A., F. LAAOUAD, K. LAROUSSI, Fuzzy Approach Applied in Fault Detection and Isolation to the Compression System C ontrol, Studies in Informatics and Control, vol 19(1), 2010.

2. BASILE, G., G. MARRO, Controlled and Conditioned Invariant Subspaces in Li near System The ory. Journal of Optimization Theory and Applications, 3, 1969. 
3. FANG, H., H. YE, M. ZHONG, Fault Diagnosis of Networked Control Systems, Annual Reviews in Control, vol. 31, 2007, pp. 55-68.

4. FRANK, P. M., Fault Dianosis in Dynamic System Using Analytical a nd Knowledge-based Red undancy -- A Survey an d Some New Resu Its. Automatica, vol. 26, 1990, pp. 459-474.

5. FRANK, P. M., X. DING, Survey of Robust Residual Generation and Evaluation Methods in Obs erver-based Fault Detection Systems . Journal of Process Control, vol. 7, 1997, pp. 403-424.

6. HWANG, I., S. KIM, Y. KIM, C. E. SEAH, A S urvey of Fault Detecti on, Isolation, and Reconfiguration Methods, IEEE Transactions on Control System Technology, vol. 18, 2010, pp. 636-653.

7. ISERMANN, R., Process Fault Detection Based on Modeling and Estim ation Methods A Survey. Automatica, vol. 4, 1984, vol. 387-404.

8. ISERMANN, R., Model-based Faultdetection and Diagnos is Status and Applications. Annual Reviews in Control, vol. 29, 2005, pp. 71-85.

9. DUZINKIEWICZ, K., A. BOROWA, K. MAZUR, M. GROCHOWSKI, K. JEZIOR, M. A. BRDYS, Leakage Detection and Localisation in Drinking Water Di stribution Network $s$ by MultiRegional PCA. Studies in Informatics and Control, vol. 17(2), 2008.

10. KIMMICH, F. Fault Detection for Modern Diesel Engines using Signal- and Process Model-based Methods*1. Control Engineering Practice, vol. 13, 2005, pp. 189-203.

11. PATTON, R. J., F. J. UPPAL, S. SIMANI, B. POLLE, Robust FDI Applied to Thruster Faults of a Satellite System, Control Engineering Practice, vol. 18, 2010, pp. 1-17.
12. GAYAKA, S., B. YAO, Output Feedback based Adaptive Robust Fault-tolerant Control for a Class of Uncertain Nonlinear Systems, Journal of Systems Engineering and Electronics, vol. 22, 2011, pp. 38-51.

13. TANWANI, A., A. D. DOMINGUEZGARCIA, D. LIBERZON, An Inversi onbased Approach to Fau It Detection and Isolation in Switching Electrical Networks. IEEE Transactions on Control Systems Technology, vol. 19, 2010, pp. 1059-1074.

14. VENKATASUBRAMANIAN, V., A Review of Process F ault Detection and Diagnosis Part I: Quantitative Model-based Methods. Computers \& Chemical Engineering, vol. 27, 2003, pp. 293-311.

15. VENKATASUBRAMANIAN, V., A Review of Process F ault Detection and Diagnosis Part II: Qualitative Models and Se arch Strategies, Computers \& Chemical Engineering, vol. 27, 2003, pp. 313-326.

16. VENKATASUBRAMANIAN, V., A Review of Process F ault Detection and Diagnosis Part III: Process History based Methods. Computers \& Chemical Engineering, vol. 27, 2003, pp. 327-346.

17. WONHAM, W. M., Linear Multivariable Control: A Geometric Approach , Applications of Mathematics, vol. 10, 3 ed., Springer, 1985.

18. XU, Y., B. JIANG, Z. GAO, K. ZHANG, Fault Tolerant Control for Near Sp ace Vehicle: A Survey and Some New Results, Journal of Systems Engineering and Electronics, vol. 22, 2011, pp. 88-94.

19. ZHANG, Y., J. JIANG, Bibliographical Review on Reconfigurable Fault-tolerant Control Systems , Annual Reviews in Control, vol. 32, 2008, pp. 229-252. 